\title{
The stability of motorcycles under acceleration and braking
}

D J N Limebeer ${ }^{1 *}$, R S Sharp ${ }^{2}$ and S Evangelou ${ }^{1}$

${ }^{1}$ Department of Electrical and Electronic Engineering, Imperial College of Science, Technology and Medicine, London, UK

${ }^{2}$ School of Mechanical Engineering, Cranfield University, Cranfield, UK

\begin{abstract}
A comprehensive study of the effects of acceleration and braking on motorcycle stability is presented. This work is based on a modified version of a dynamic model presented earlier, and is thought to be the most comprehensive motorcycle dynamic model in the public domain. Extensive use is made of both non-linear and linearized models. The models are written in LISP and make use of the multibody modelling package AUTOSIM. There is novelty in the way in which control systems have been used to control the motorcycle drive and braking systems in order that the machine maintains desired rates of acceleration and deceleration.

The results show that the wobble mode of a motorcycle is significantly destabilized when the machine is descending an incline or braking on a level surface. Conversely, the damping of the wobble mode is substantially increased when the machine is ascending an incline at constant speed, or accelerating on a level surface. This probably accounts for the pleasingly stable 'feel' of the machine under firm acceleration.

Except at very low speeds, inclines, acceleration and deceleration appear to have little effect on the damping or frequency of the weave mode. Non-linear simulations have quantified the known difficulties to do with rear tyre adhesion in heavy braking situations that are dominated by rear wheel braking.
\end{abstract}

Keywords: motorcycle stability, wobble, weave, mathematical modelling, multibody analysis, stoppie, wheelie

\section{NOTATION}

$a \quad$ target acceleration $\left(\mathrm{m} / \mathrm{s}^{2}\right)$

drive total externally applied wheel moment $(\mathrm{N} \mathrm{m})$

$\boldsymbol{F}_{\text {check }}$ longitudinal saturation force check (N)

$\boldsymbol{F}_{\text {drag }}$ aerodynamic drag force vector $(\mathrm{N})$

$\boldsymbol{F}_{\text {lift }} \quad$ aerodynamic lift force vector $(\mathrm{N})$

$\boldsymbol{F}_{\text {load }}$ normal tyre load $(\mathrm{N})$

$\boldsymbol{F}_{\text {long }}$ longitudinal tyre force $(\mathrm{N})$

$\boldsymbol{F}_{\text {rear }} \quad$ rear longitudinal tyre force $(\mathrm{N})$

$M_{\text {front }}$ moment applied to the front wheel $(\mathrm{N} \mathrm{m})$

$M_{\text {rear }}$ moment applied to the rear wheel $(\mathrm{N} \mathrm{m})$

$P_{\text {aero }} \quad$ power loss due to aerodynamic effects $(\mathrm{kW})$

$P_{\text {rear }} \quad$ power supplied at the rear wheel $(\mathrm{kW})$

$\begin{array}{ll}P_{\text {tyre }} & \text { power loss in rear tyre }(\mathrm{kW}) \\ s_{\text {rear }} & \begin{array}{l}\text { longitudinal slip of rear tyre } \\ t\end{array} \\ v_{\text {i }} & \begin{array}{l}\text { time }(\mathrm{s}) \\ v_{\text {main }}\end{array} \\ v_{\text {rear }} & \begin{array}{l}\text { velocity of rear frame assembly }(\mathrm{m} / \mathrm{s}) \\ \text { velocity of rear tyre contact point projected } \\ \text { on to the line of intersection between the }\end{array} \\ & \begin{array}{l}\text { wheel plane and the ground plane }(\mathrm{m} / \mathrm{s}) \\ \text { speed reference }(\mathrm{m} / \mathrm{s})\end{array} \\ v_{\text {ref }} & \begin{array}{l}\text { braking distribution constant } \\ \lambda\end{array} \\ \omega_{\text {rear }} & \text { angular velocity of the rear wheel }(\mathrm{rad} / \mathrm{s})\end{array}$

\section{INTRODUCTION}

Virtually all the published work addressing the stability of motorcycles has focused on the constant forward
The MS was received on 9 February 2001 and was accepted after revision for publication on 14 March 2001.

*Corresponding author: Department of Electrical and Electronic Engineering, Imperial College of Science, Technology and Medicine, Exhibition Road, London SW7 2BT, UK. 
speed case, with the bulk of this research concentrating on the vehicle's lateral dynamic stability under straight running conditions (see references [1] to [5] and the many references therein). Readers who are interested in the historical development of this area of research are referred to the comprehensive survey article [6]. It can be seen from this paper that the early literature modelled the vehicle using simple rigid body representations for the front and rear frames, while the road-tyre rolling contact was treated as a longitudinal rolling constraint. Over time, this sequence of models treated the tyres as more and more sophisticated moment and force producers, and they also evolved to include the effects of various frame flexibilities and rider dynamics. The case of constant-speed cornering is considerably more complicated to model and it has consequently received much less attention in the classical literature [7] - the literature prior to 1990. The dynamic properties of single-track vehicles under acceleration and braking has also remained relatively under studied, and as far as the present authors are aware, the only work in this area is that given in reference [8]. This study employed the analysis techniques introduced in the context of the jack-knifing of articulated vehicles [9]. By contemporary standards, this study is somewhat simplistic, because it uses a simple vehicle model and it only considers the 'inertial effects' of acceleration. Also, the influences of acceleration on the tyre loads were not treated accurately, because the model did not include a suspension system.

As multibody modelling codes such as AUTOSIM [10] became available, it has become possible to treat far more complicated situations, such as stability under cornering $[\mathbf{1 1}, \mathbf{1 2}]$ and vehicles with dynamically variable rear suspension geometries [13]. These new and powerful computer-assisted modelling tools have also made it possible to re-examine problems such as vehicle stability under braking and acceleration in far more detail. The main reason for these advances is that computer codes have removed the insurmountable fatigue barriers associated with tedious and complex manual multibody model-building exercises. To make matters worse, even if one survives this process, it is nigh on impossible to qualify adequately manually built models and ensure that they are error free.

The goal of the present study is to re-examine the straight running stability of motorcycles under braking and acceleration. In order to do this, use will be made of an evolution of the foundation AUTOSIM model described in reference [12]. Interested readers may also wish to consult the reports [14] and [15] that cover the development of relatively simple linear and non-linear motorcycle models in the AUTOSIM multibody framework-these models have been qualified against the prior art.

The basic components of the motorcycle model used here are: (a) separate bodies for the front and rear frame that are joined by an inclined steering axis freedom; (b) a rear frame that is allowed longitudinal, lateral and heave translational freedoms, as well as yaw, pitch and roll angular freedoms; (c) spinning road wheels; (d) twist and steer freedoms for the rear frame relative to the front frame; (e) a steady state tyre force and moment system that allows longitudinal slip which is based on the ideas in references [7] and [16]; (f) introduction of first-order lags to represent tyre relaxation effects, whereby the tyre forces and moments do not respond immediately to changes in the tyre slip; $(\mathrm{g})$ inclusion of simple aerodynamic effects, so that the tyre loads respond properly to changes in speed; (h) a freedom that allows the rider's body to roll with respect to the motorcycle's rear frame; (i) suspension systems.

It is now widely accepted that the straight running stability of motorcycles is dominated by three modes: the capsize, weave and wobble modes. The capsize mode relates to a slow roll dynamic that can be either stable, or slightly unstable with a time constant of 5$10 \mathrm{~s}$. This mode is relatively easily dealt with by the rider and is therefore of no great importance for present purposes. The weave mode comprises a 'fish tailing' motion that is a combination of roll and yaw of the whole vehicle-rider system. This mode is generally stable, but can become unstable and even dangerous under certain combinations of operating conditions, vehicle configuration and rider control actions. It is the belief of the authors that this mode of behaviour is still not fully understood. The wobble mode involves primarily a $6-10 \mathrm{~Hz}$ oscillation of the fork assembly relative to the main frame of the machine. The frequency of this mode is relatively insensitive to speed variations and its damping is affected by parameters such as the trail geometry, steering dampers and the nature of the rider's grip on the handle bars.

Figure 1 shows these modes in the constant speed case over the range $0.1-67.9 \mathrm{~m} / \mathrm{s}$ and is a plot of the speeddependent eigenvalues of the linearized motorcycle model. The wobble mode covers the frequency range $6.4-8.0 \mathrm{~Hz}$, while the maximum weave mode frequency is approximately $3.8 \mathrm{~Hz}$. The rear and front suspension bounce modes can also be identified with frequencies of 2.55 and $1.55 \mathrm{~Hz}$ respectively. As explained in reference [14], the weave mode starts out (at a very low speed) as two distinct real unstable modes that are marked as a and $b$ in Fig. 1. The a mode corresponds to the body capsize mode, while the $\mathrm{b}$ mode corresponds to the steering capsize mode [14]. As the machine speed increases, these roots move closer together, coalesce and then form the weave mode. The behaviour of the wobble and weave modes under acceleration and deceleration is the main focus of this paper.

The mathematical models used to generate the present results are described in Section 2. Included is a description of the drive and braking moment models and the checks used to qualify the AUTOSIM models. Section 3 


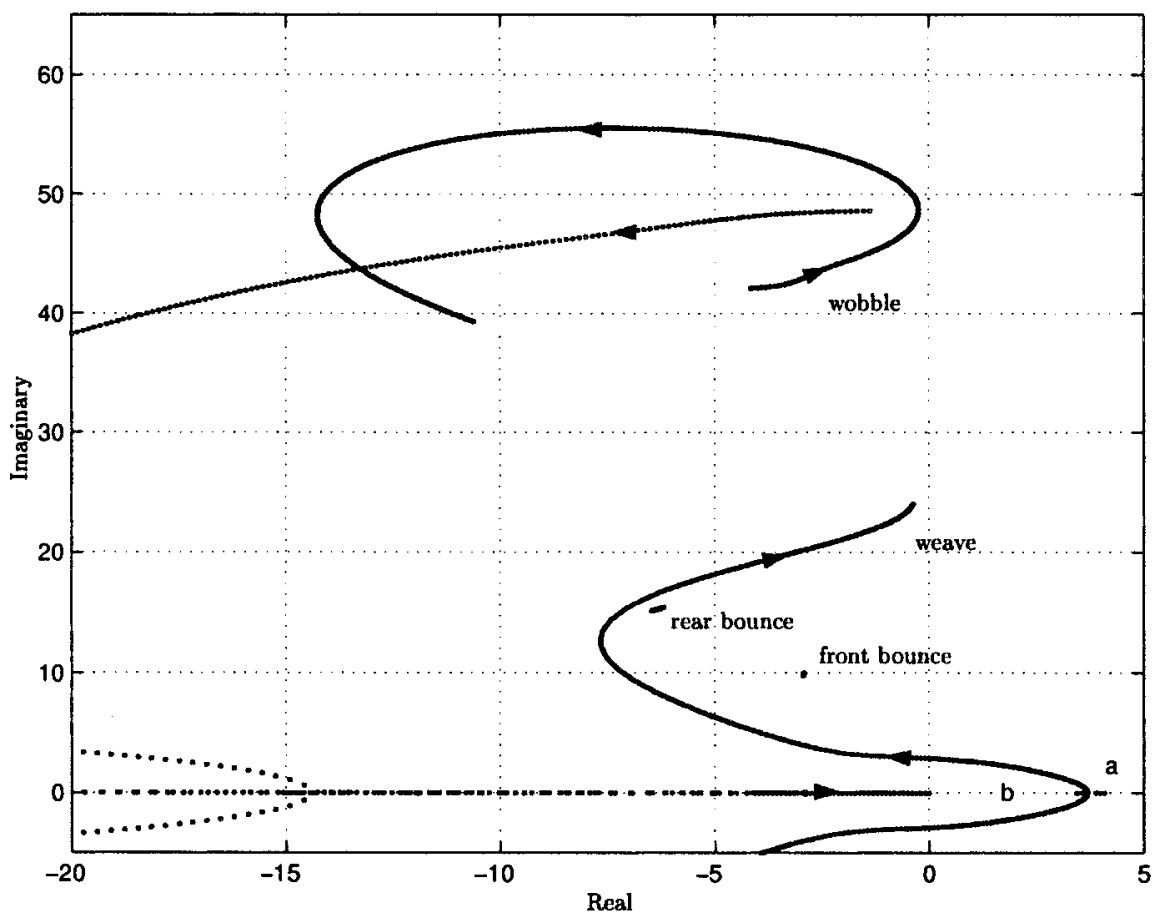

Fig. 1 Root locus with speed the varied parameter. The speed is increased from 0.1 to $67.9 \mathrm{~m} / \mathrm{s}$ in the direction of the arrows

provides a brief review of relevant stability theory for linear time-varying systems. The main findings of the work are given in Section 4 and the conclusions appear in Section 5.

\section{THE MATHEMATICAL MODEL}

The motorcycle model employed in this paper is based on that presented in Section 3 of reference [12]. The description given here is only a brief summary of this previous work and should therefore be read in conjunction with it. Figure 2 shows the machine in its nominal configura- tion in static equilibrium with the key modelling points labelled as $p_{1}, \ldots, p_{14}$. The symbolic multibody modelling package AUTOSIM [10] is used to convert the conceptual model into a FORTRAN code that is used to facilitate the non-linear simulation studies, or MATLAB M-files in the case of linearized model-based studies. The linearized models are used in stability studies of the frozen-time eigenvalues type. The childparent structure used here is very similar to that employed in reference [12] and is reproduced in Fig. 3 for ease of reference.

The model contains the following components: a main frame, a swinging arm and its associated rear suspension system, a body with a roll freedom relative to the main

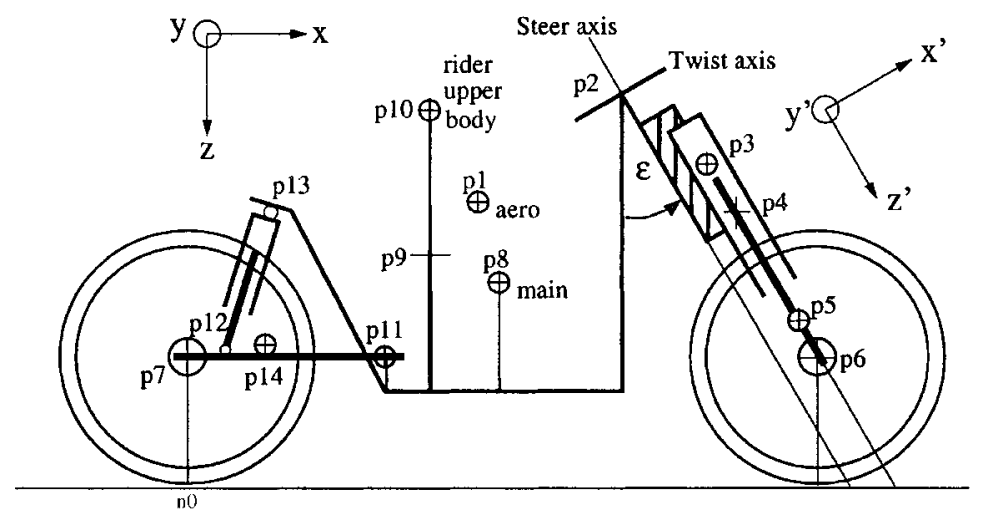

Fig. 2 Motorcycle model in its nominal configuration 


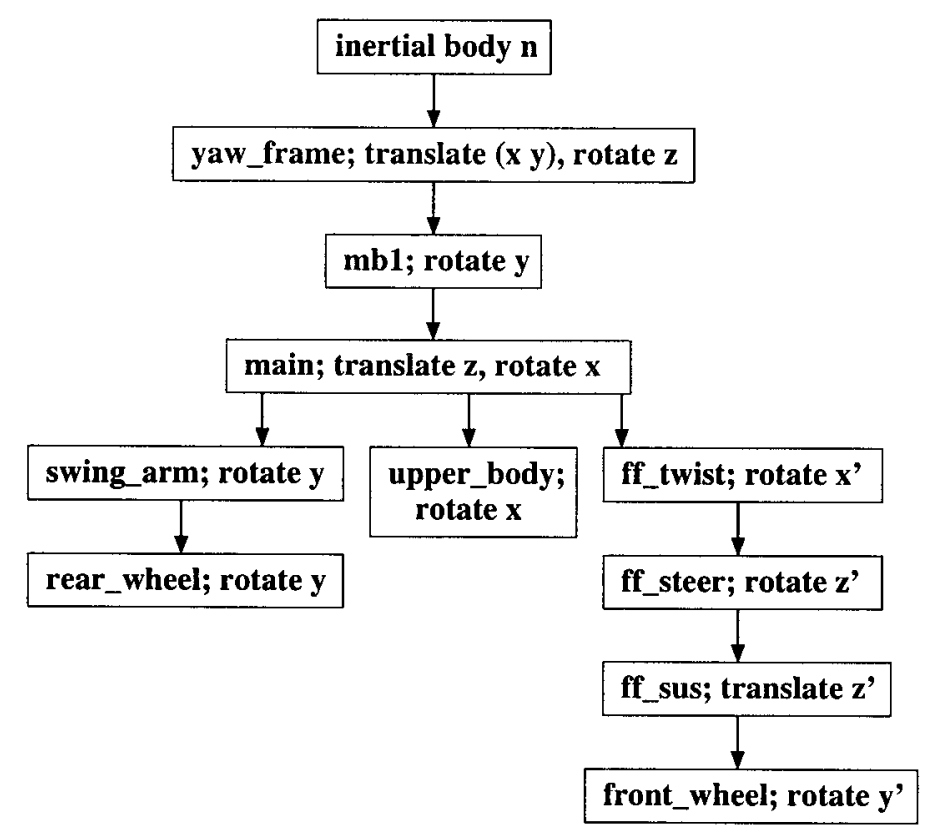

Fig. 3 Body structure diagram showing the freedoms and the parent-child relationships

frame that is used to represent the upper body of the rider, a front frame with twist and steer freedoms, telescopic front forks, spinning road wheels and dynamic tyres. The road is assumed to be flat and the motorcycle can travel anywhere in the horizontal plane. The longitudinal tyre forces are proportional and opposed to the longitudinal slip as defined in reference [16]. Checks are made to ensure that these longitudinal tyre forces do not exceed 80 per cent of the normal tyre load. If this figure is exceeded by either tyre at any point during a simulation run, then the results are deemed invalid because they lie outside the tyre model's intended operating regime. The longitudinal tyre forces are assumed to be decoupled from the lateral tyre forces and moments, which are computed using the empirical expressions given in reference [7]. Relaxation effects are associated with the lateral force systems, but not with the longitudinal ones. The wheel-aligning moments due to side-slip include relaxation effects, while those due to camber are assumed to be instantaneous. The overturning moments due to camber are assumed to be instantaneous functions of the tyre contact geometry $[\mathbf{1 1}, \mathbf{1 2}]$. In-plane aerodynamic forces and moments are also included.

Each wheel-tyre combination is treated as a thin disc with a radial flexibility freedom. The massless outer ring of the tyre can translate from contact point to wheel centre, with a spring force restraint used to represent the tyre wall compliance. The complex dynamic geometry associated with the migration of the tyre contact points (especially that of the front tyre) is an outstanding feature of this model and is described elsewhere $[11,12]$.

\subsection{Drive and braking moments}

The drive and braking moments were generated using proportional plus integral control signals and are based on a speed error-the difference between the actual speed and a speed reference signal. In most cases the speed reference was a simple ramp function of the form:

$$
v_{\text {ref }}=v_{\mathrm{i}}+a t
$$

When the applied wheel moment is a (negative) drive moment it is applied to the rear wheel alone. In the case of a (positive) braking moment, it is split in the ratio $(1-\lambda): \lambda$ between the rear and front wheels respectively. The constant $\lambda$ is given by $\lambda=0.9$ for predominantly front wheel braking and $\lambda=0.1$ for predominantly rear wheel braking. In order to implement these ideas, the driving/braking moment applied to the rear wheel is computed via

$$
M_{\text {rear }}=\min (\text { drive, drive } *(1-\lambda))
$$

while that applied to the front wheel is

$$
M_{\text {front }}=\max (0.0, \text { drive } * \lambda)
$$

For further details, the reader is referred to the AUTOSIM code that can be found at the following web site: http://www.ee.ic.ac.uk/control/motorcycles. This code contains a lot of fine detail that is only discussed briefly, or is not discussed at all. The code also contains a complete list of the motorcycle parameters; most, but not all, are the same as those used in reference [12]. 


\subsection{Model checking}

Given the complexity of the simulation model, it is necessary to take considerable care with the model validation process. Where appropriate, use is made of the equilibrium checks described in Section 4 of reference [12].

Since the bulk of the studies described here are acceleration/braking investigations, equilibrium tests are usually inappropriate. It was therefore necessary to introduce a power balance test that ensures that all the power applied to the rear wheel is accounted for. The power developed at the rear wheel is simply

$$
P_{\text {rear }}=M_{\text {rear }} \omega_{\text {rear }}
$$

The power dissipated by the aerodynamic forces is:

$$
P_{\text {aero }}=-\operatorname{dot}\left[\left(\boldsymbol{F}_{\text {drag }}+\boldsymbol{F}_{\text {lift }}\right), \boldsymbol{v}_{\text {main }}\right]
$$

in which $\operatorname{dot}(\cdot, \cdot)$ represents a dot product between the indicated vectors. Finally, the power dissipated in the rear tyre is

$$
P_{\text {tyre }}=-\boldsymbol{F}_{\text {rear }} v_{\text {rear }} S_{\text {rear }}
$$

The check is that these three power terms sum to zero. This check was carried out and proved successful for all the results presented here.

\section{STABILITY/INSTABILITY OF TIME-VARYING SYSTEMS}

Mathematical models of motorcycles under acceleration and decelerating are time-varying systems and so are their dynamic stability properties. The purpose of this short section is to review briefly some of the stability/ instability properties of time-varying systems.

It is well known that the $n$ th-order differential equation

$$
\dot{\boldsymbol{x}}(t)=\mathbf{A} \boldsymbol{x}(t), \quad \boldsymbol{x}(0)=\boldsymbol{x}_{0}
$$

has the solution

$$
\boldsymbol{x}(t)=\sum_{i=1}^{n} \boldsymbol{w}_{i} \mathrm{e}^{\lambda_{i} t} \boldsymbol{v}_{i}^{*} \boldsymbol{x}_{0}
$$

in which the $\lambda_{i}$ s are the eigenvalues of $\mathbf{A}$ (for simplicity $\mathbf{A}$ is assumed to be diagonalizable) and the $\boldsymbol{w}_{i}$ s and $\boldsymbol{v}_{i}$ s are the corresponding eigenvectors and dual eigenvectors respectively. These solutions will vanish asymptotically if $\operatorname{Re}\left(\lambda_{i}\right)<0$. In other words, for an arbitrary $\boldsymbol{x}_{0}$ the solutions of this equation are stable if (and only if) all the eigenvalues of $\mathbf{A}$ have negative real parts. In general, the stability properties of linear time-varying systems cannot be tested using the eigenvalues in this way. For example, the matrix

$$
\mathbf{A}(t)=\left[\begin{array}{cc}
-1 & \mathrm{e}^{2 t} \\
0 & -1
\end{array}\right]
$$

has both its eigenvalues at -1 for all $t$, but the corresponding system $\dot{\boldsymbol{x}}(t)=\mathbf{A}(t) \boldsymbol{x}(t)$ is unstable in the sense that for some initial conditions $\lim _{t \rightarrow \infty} \boldsymbol{x}(t)$ is unbounded [17]. Therefore, in general, there is no significance to the concept of a 'mode', or a 'time-varying natural frequency' in the case of time-variant linear systems. Consider

$$
\dot{\boldsymbol{x}}(t)=\mathbf{A}(t) \boldsymbol{x}(t), \quad \boldsymbol{x}_{0}=\boldsymbol{x}(0)
$$

Provided $\dot{\mathbf{A}}(t)$ is small enough for all $t \geqslant 0$, it would be expected intuitively that the time-varying system will be stable provided that for each frozen time $\bar{t}$, the (frozentime) system $\mathbf{A}(\bar{t})$ is stable. It is known [18] that if the eigenvalues of $\mathbf{A}(t)$ have real parts that are sufficiently negative for all $t \geqslant 0$ and $\sup _{t \geqslant 0}\|\dot{\mathbf{A}}(t)\|$ is sufficiently small, then the solutions of $\dot{\boldsymbol{x}}(t)=\mathbf{A}(t) \boldsymbol{x}(t)$ go to zero as $t \rightarrow \infty$.

There might also be trouble when predicting instability using the frozen-time eigenvalues of $\mathbf{A}(t)$. If $\mathbf{A}(t)$ has at least one frozen-time eigenvalue with positive real part, the solutions of $\dot{\boldsymbol{x}}(t)=\mathbf{A}(t) \boldsymbol{x}(t)$ may be stable. One would expect that if $\mathbf{A}(t)$ has eigenvalues in the right half plane, then the system $\dot{\boldsymbol{x}}(t)=\mathbf{A}(t) \boldsymbol{x}(t)$ will have unbounded solutions if $\sup _{t \geqslant 0}\|\dot{\mathbf{A}}(t)\|$ is sufficiently small. This is indeed the case provided no eigenvalue crosses the imaginary axis [19]. If eigenvalues are allowed to cross the imaginary axis, then even though there is always an eigenvalue with positive real part, the system can be asymptotically stable for arbitrarily small $\sup _{t \geqslant 0}\|\dot{\mathbf{A}}(t)\|$. Consider the matrix [19]

$$
\mathbf{A}(t)=\left[\begin{array}{cc}
-1+\alpha \cos \omega t \sin \omega t & \alpha \cos ^{2} \omega t+\omega \\
-\alpha \sin ^{2} \omega t-\omega & -1-\alpha \cos \omega t \sin \omega t
\end{array}\right]
$$

The corresponding transition matrix $\phi\left(t, t_{0}\right)$ is given by

$$
\begin{aligned}
\Phi\left(t, t_{o}\right)= & \mathrm{e}^{-\left(t-t_{0}\right)}\left[\begin{array}{cc}
\cos \omega t & \sin \omega t \\
-\sin \omega t & \cos \omega t
\end{array}\right]\left[\begin{array}{cc}
1 & \alpha\left(t-t_{0}\right) \\
0 & 1
\end{array}\right] \\
& \times\left[\begin{array}{cc}
\cos \omega t_{0} & -\sin \omega t_{0} \\
\sin \omega t_{0} & \cos \omega t_{0}
\end{array}\right]
\end{aligned}
$$

and so with this $\mathbf{A}(t)$ all the corresponding equation solutions are exponentially bounded. It is easy to check that the eigenvalues of $\mathbf{A}(t)$ are time independent and given by

$$
\lambda=-1 \pm \sqrt{-\alpha \omega-\omega^{2}}
$$

Setting $\omega=1$ and $\alpha=-5$, the eigenvalues of $\mathbf{A}(t)$ are at +1 and -3 for all time. Note, however, that for any 
$\alpha<-2$, if

$$
0<\omega<-\frac{\alpha}{2}-\frac{1}{2} \sqrt{\alpha^{2}-4}
$$

or

$$
\omega>-\frac{\alpha}{2}+\frac{1}{2} \sqrt{\alpha^{2}-4}
$$

then the eigenvalues of $\mathbf{A}(t)$ have negative real parts. Thus, when $\mathbf{A}(t)$ is varying either slowly or rapidly, the eigenvalues of $\mathbf{A}(t)$ correctly predict the stability properties of the system. When $\omega$ lies between the aforementioned limits, they do not.

The idea of a 'mode' will be used for the linear timevarying systems and the eigenvalues of frozen-time linearized models will be used to infer stability properties, but it is recognized that this must be done with great caution.

\section{RESULTS}

Root-loci and non-linear simulation results are presented that show the effects of acceleration and deceleration on motorcycle stability. The main emphasis will be on the weave and wobble modes, as these are the dominant ones under the acceleration/deceleration conditions of interest here. The non-linear simulation results come directly from the FORTRAN simulation codes generated by AUTOSIM. The root-locus plots are generated via the eigenvalues of frozen-time symbolic linearized state-space models (also generated by AUTOSIM). The evaluation of the linearized state-space model matrices requires information about the frozen-time values of the various model states - this information is provided by the non-linear simulation codes. In order to generate a root-locus plot, the non-linear simulation model is accelerated/decelerated over the speed range of interest. These data had to be checked to ensure: (a) that the rear wheel did not leave the ground (thereby producing a stoppie); (b) that the front wheel did not leave the ground (thereby producing a wheelie); (c) that the tyres did not undergo longitudinal saturation; and (d) that the engine power did not exceed $65 \mathrm{~kW}$. The saturation condition was checked via the negativity, or otherwise, of the test force:

$$
\boldsymbol{F}_{\text {check }}=0.8 \boldsymbol{F}_{\text {load }}+\left|\boldsymbol{F}_{\text {long }}\right|
$$

in which $\boldsymbol{F}_{\text {load }}$ is always negative, while $\boldsymbol{F}_{\text {long }}$ can be positive or negative. If $\boldsymbol{F}_{\text {check }} \geqslant 0$, the tyre was deemed to have saturated and the associated simulation data was disregarded. The root-locus plots that correspond to the constant-speed cases were generated by accelerating the machine gradually over the speed range of interest.

\subsection{Straight running on an incline}

The results in this paper begin by building on the intuitive ideas in references [8] and [9]. To do this, the stability properties of the machine on inclined surfaces are studied at constant speed. The idea is that ascending/descending inclined running surfaces generates gravitational forces that mimic the inertial forces associated with acceleration/deceleration conditions, respectively. It should also be noted that the constant-speed condition means that there is no temporal variation in the aerodynamic loading as the speed changes and that the associated linearized models are time-invariant. There is therefore no need to consider the complications associated with the stability testing, via the eigenvalues of frozen-time linearized models, of time-varying systems. Figure 4 shows root-locus plots for the cases of straight running, at constant speed, on both level and inclined smooth surfaces. The speed ranges associated with the various cases are dictated by the limiting conditions alluded to above. The first thing to note is that the wobble mode is destabilized significantly on downhill (as opposed to uphill) inclinations. It can also be seen that the wobble mode is marginally more stable under rear-wheel-dominated braking. It is the common wisdom that one should use rear-wheel-dominated braking on downhill slopes at very low speeds, especially in slippery road conditions. Figure 4 also shows that inclined road surfaces have very little influence on the weave mode. At very low speeds, the weave mode remains real over a slightly greater range of speeds under front-wheel-dominated braking. Intuitively, this makes the machine harder to control under these conditions, because it tends to just 'fall over', rather than undergo an unstable lowfrequency 'drunkard's walk'. This could be the reason for the rider training advice: 'At very low speed control the machine by balancing the throttle and rear brake'.

\subsection{Acceleration studies}

Figures 5, 6 and 7 consider the effects of acceleration on motorcycle stability. As expected, the general trends follow those associated with the results obtained for constant speeds on ascending slopes. The reason for this is that the inertial forces act in the same direction in the acceleration case as the gravity forces do in the uphill case. Figure 5 shows that the weave mode is hardly affected by the effects of acceleration, while the wobble mode is substantially more heavily damped. These effects probably account for the good 'feel' associated with powerful machines under firm acceleration. In the bar room biker's vernacular: 'it feels as if it is running on rails'.

Figure 6 shows the effect of speed on the aerodynamic drag, the tyre loads, the drive torque and the front tyre saturation. As expected, the aerodynamic drag increases 


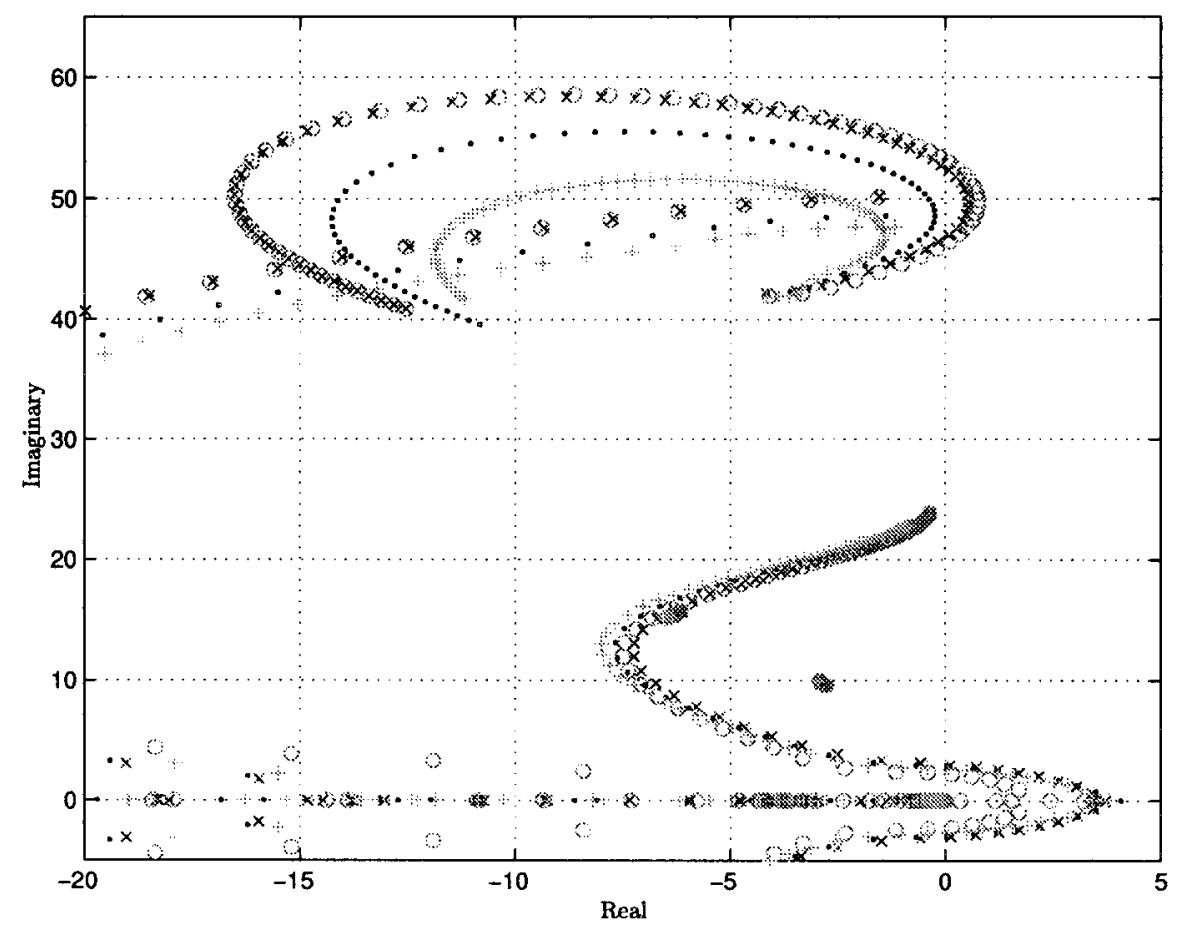

\begin{tabular}{lclll}
\hline Plot symbol & Inclination angle (rad) & Front brake $(\%)$ & Rear brake $(\%)$ & Speed range $(\mathrm{m} / \mathrm{s})$ \\
\hline$\cdot$ & 0 & - & - & $0.1-67.9$ \\
$\times$ & -0.2 & 10 & 90 & $0.06-70.0$ \\
$\circ$ & -0.2 & 90 & 10 & $0.06-70.0$ \\
+ & 0.2 & - & - & $0.02-53.0$ \\
\hline
\end{tabular}

Fig. 4 Root loci for straight running on level and inclined smooth surfaces. Positive inclination angles correspond to the uphill case, whereas negative ones correspond to the downhill case

quadratically with speed, as does the required drive moment. The aerodynamic drag also tends to load the rear wheel, while correspondingly lightening the normal load on the front tyre. Also, as expected, the increased drive torque and longitudinal tyre force bring the rear tyre closer to saturation.

In Section 3 the reader was reminded that the stability of linear time-varying systems cannot be tested using the frozen-time eigenvalues of $\mathbf{A}(t)$ alone. With that warning in mind, the transient behaviour of the machine was examined with the non-linear simulation model and compared with the outcomes predicted by the results given in Fig. 5. By re-examining that plot it can be seen that the weave mode of the frozen-time model is unstable at time $t_{1}$, neutrally stable at $t_{2}$ and stable at $t_{3}$. The goal is to check that the non-linear simulation model reproduces, qualitatively, that same behaviour. Given the approximations involved, it is unrealistic to expect exact quantitative agreement. Figure 7 shows the response of the non-linear model to a steering angle offset of $0.1 \mathrm{rad}$ at the unstable initial time $t_{1}$. This plot shows that an unstable behaviour builds up, and then decays over the time interval $t_{1}$ to $t_{2}$. This temporary growth appears to be dominated by the weave mode and, as predicted by the frozen-time model, dies out by the time $t_{2}$ is reached. As far as the weave mode is concerned, in this case the frozen-time linear model appears to be pessimistic in its predictions.

\subsection{Deceleration studies}

It has already been seen that downhill running tends to destabilize the wobble mode, while the weave mode remains relatively unaffected. One expects to see these trends reproduced in the deceleration studies, because the inertial forces in deceleration are equivalent to the gravitational forces in the downhill case. Figure 8 shows that these expectations are substantially true. It can be seen from this figure that the wobble mode becomes significantly less stable under braking and the effects become exaggerated as the deceleration rate increases. This figure also shows that the weave mode remains relatively unaffected by braking - as with the downhill case, the weave mode is affected most at very low speed. Figure 9 shows the anticipated changes in 


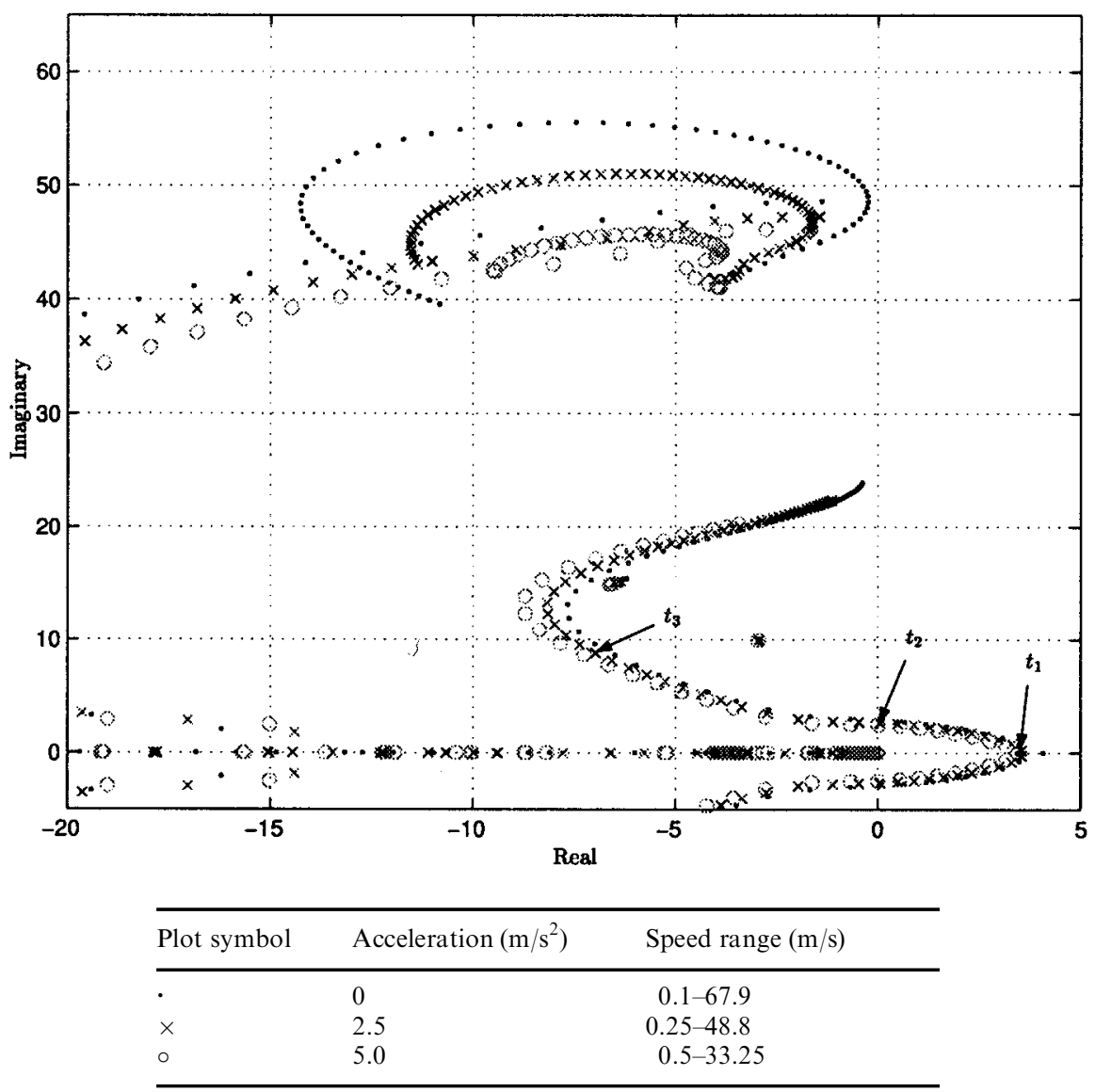

Fig. 5 Root loci for constant speed and steady acceleration on a level surface

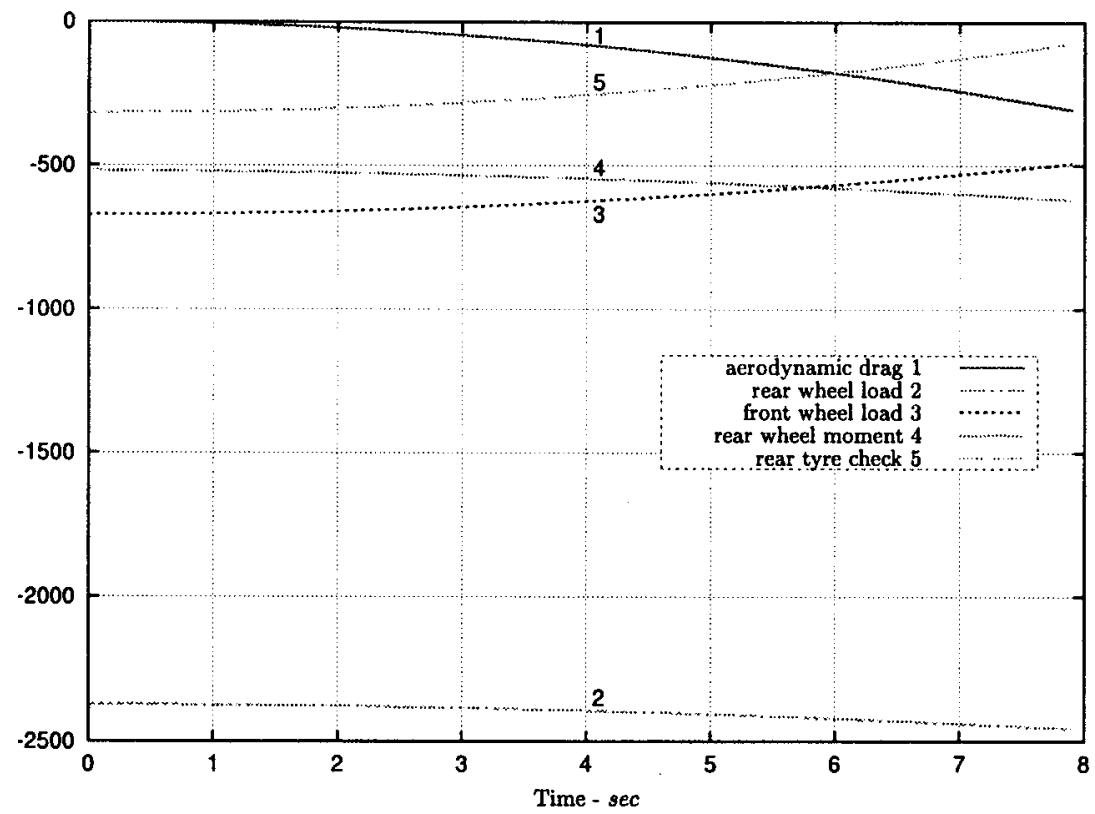

Fig. 6 The wheel loads, the rear wheel drive moment, the aerodynamic drag and the rear wheel longitudinal tyre force check for the $5 \mathrm{~m} / \mathrm{s}^{2}$ acceleration case. All the forces are given in $\mathrm{N}$, while the moment has units of $\mathrm{N} \mathrm{m}$. The tyre force-check curve is also given in $\mathrm{N}$ 


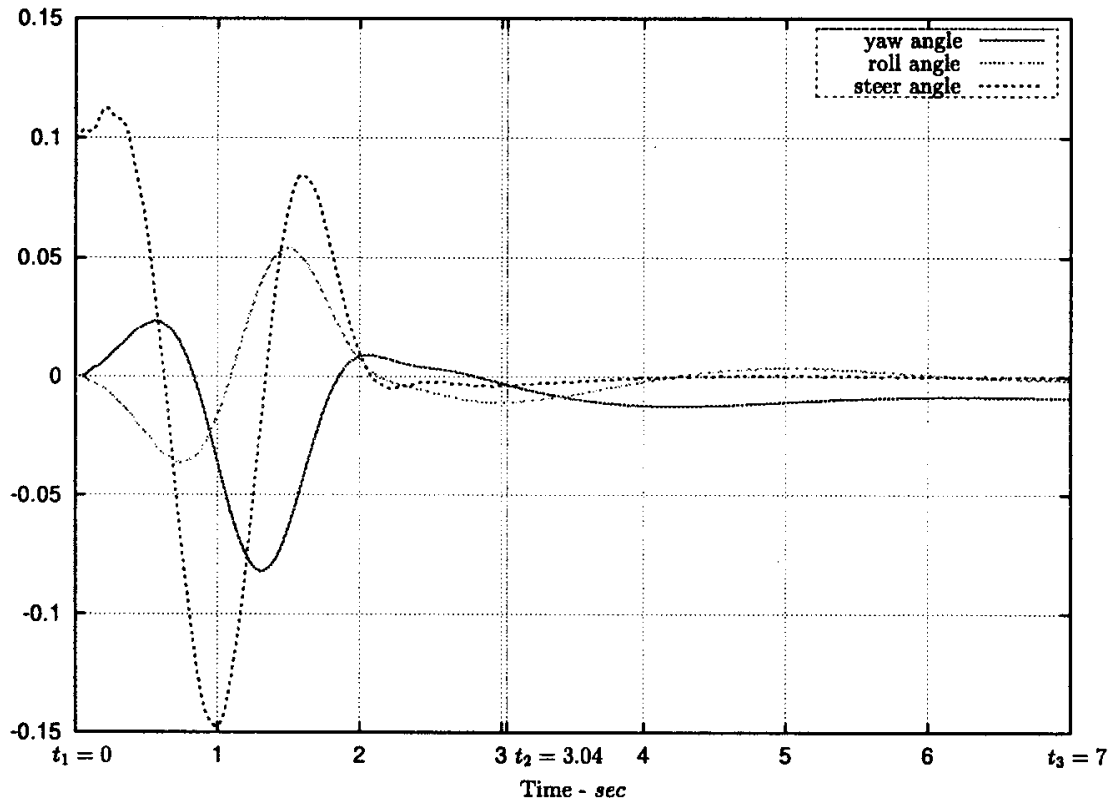

Fig. 7 Transient response of the weave mode for the $2.5 \mathrm{~m} / \mathrm{s}^{2}$ acceleration case. The initial speed is $0.25 \mathrm{~m} / \mathrm{s}$ and the initial steer angle offset is $0.1 \mathrm{rad}$; the speed at $t_{2}$ is $7.85 \mathrm{~m} / \mathrm{s}$, while that at $t_{3}$ is $17.75 \mathrm{~m} / \mathrm{s}$. The time origin corresponds to the point $t_{1}$ in Fig. 5, and the other two time-marker points are labelled as $t_{2}$ and $t_{3}$

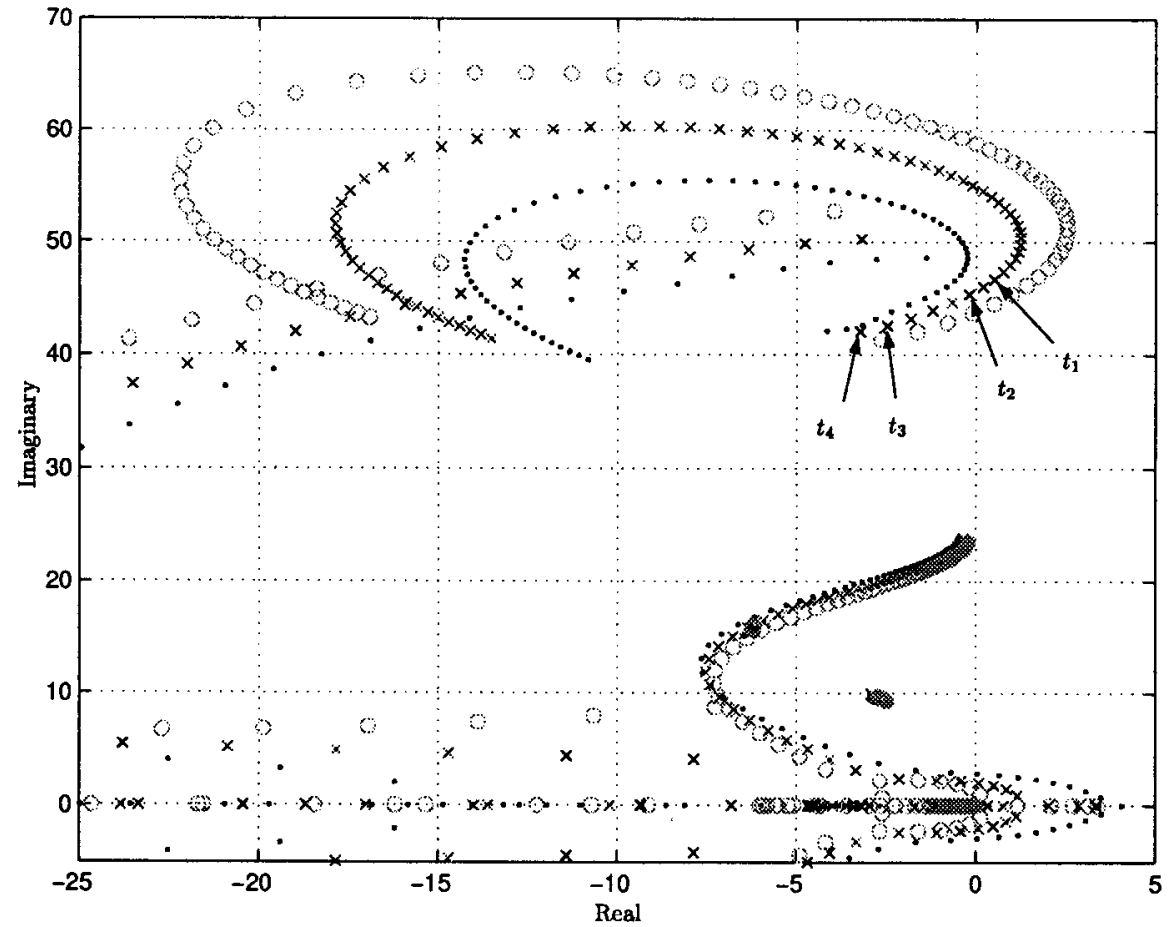

\begin{tabular}{lllll}
\hline Plot symbol & Deceleration $\left(\mathrm{m} / \mathrm{s}^{2}\right)$ & Front brake $(\%)$ & Rear brake $(\%)$ & Speed range $(\mathrm{m} / \mathrm{s})$ \\
\hline$\cdot$ & 0 & - & - & $0.1-67.9$ \\
$\times$ & 2.5 & 90 & 10 & $70.0-0.126$ \\
$\circ$ & 5 & 90 & 10 & $70.0-0.8$ \\
\hline
\end{tabular}

Fig. 8 Root loci for constant speed straight running and steady rates of deceleration. A level surface is used throughout. Note the four time markers labelled $t_{1}$ to $t_{4}$ 


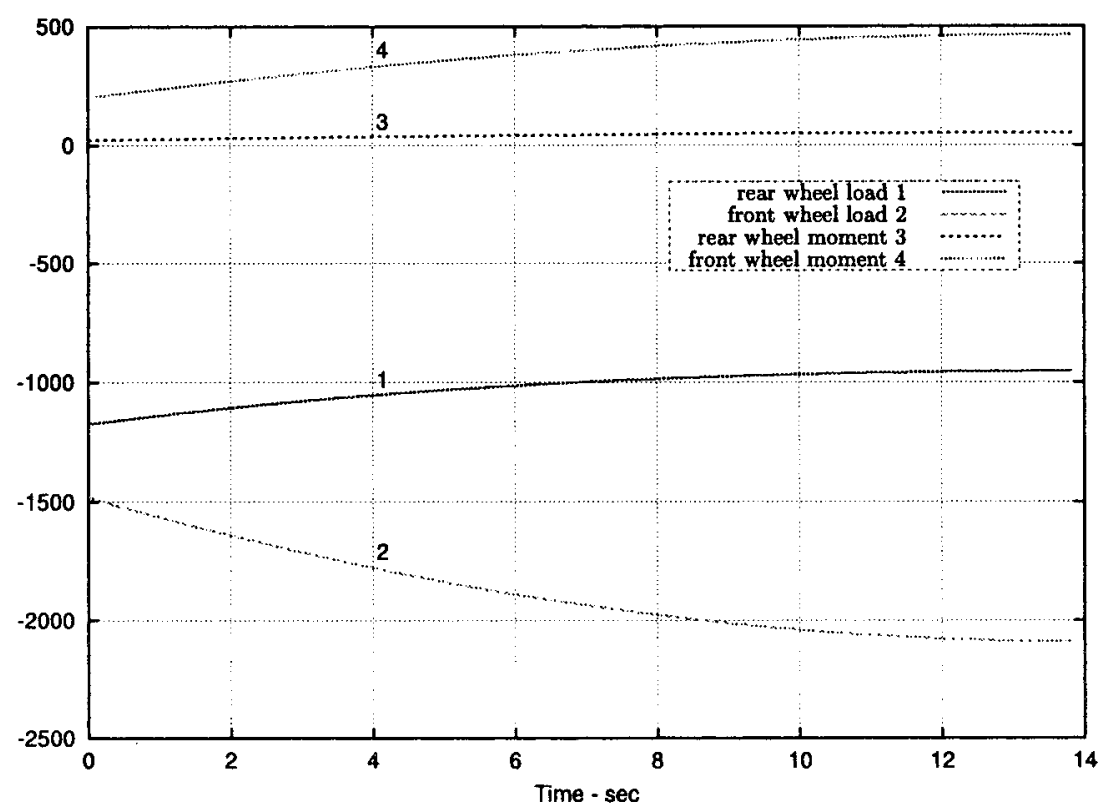

Fig. 9 The normal wheel loads and drive/braking moments in the $5 \mathrm{~m} / \mathrm{s}^{2}$ deceleration case. The braking strategy is 90 per cent on the front wheel and 10 per cent on the rear. All the forces are given in N, while the moments have units of $\mathrm{N} \mathrm{m}$

the wheel loads and wheel drive moments under braking. As expected, the bulk of the motorcycle's weight is carried by the front wheel, as is the bulk of the braking torque (under front-wheel-dominated braking). Note how the braking moment increases as the speed drops. This is explained by the fact that the aerodynamic drag does most of the high-speed braking, but this task is then taken over by the brakes as the aerodynamic drag reduces. Figure 10 is used to check the stability interpretations being given to the root loci in Fig. 8. As the speed decreases, the $2.5 \mathrm{~m} / \mathrm{s}^{2}$ wobble mode moves through the time markers $t_{1}, t_{2}, t_{3}$ and $t_{4}$ in that order.

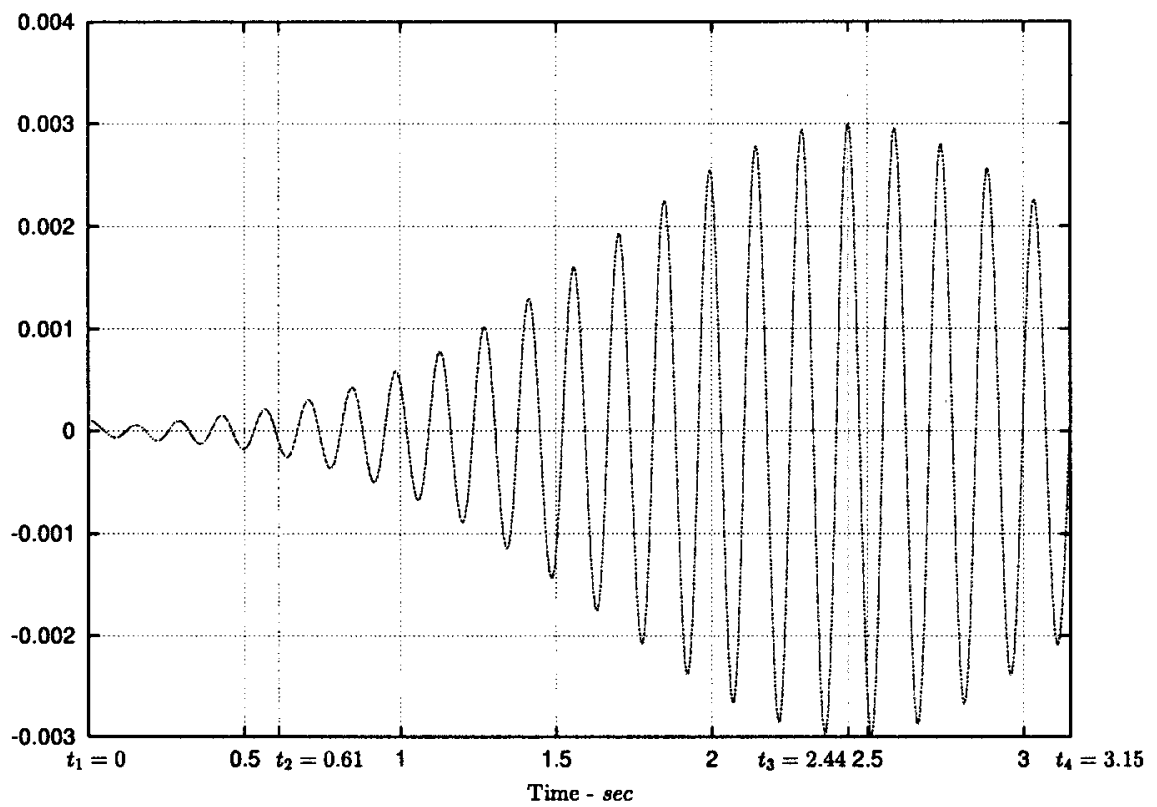

Fig. 10 Transient response of the steering angle in the $2.5 \mathrm{~m} / \mathrm{s}^{2}$ deceleration case. The initial speed is $8 \mathrm{~m} / \mathrm{s}$ and the initial steer angle offset is $0.0001 \mathrm{rad}$, the speed at $t_{1}$ is $8 \mathrm{~m} / \mathrm{s}$, the speed at $t_{2}$ is $6.48 \mathrm{~m} / \mathrm{s}$, the speed at $t_{3}$ is $1.9 \mathrm{~m} / \mathrm{s}$, while that at $t_{4}$ is $0.13 \mathrm{~m} / \mathrm{s}$. The time origin corresponds to the point $t_{1}$ in Fig. 8 , while the other three time-marker points are labelled $t_{2}, t_{3}$ and $t_{4}$ 


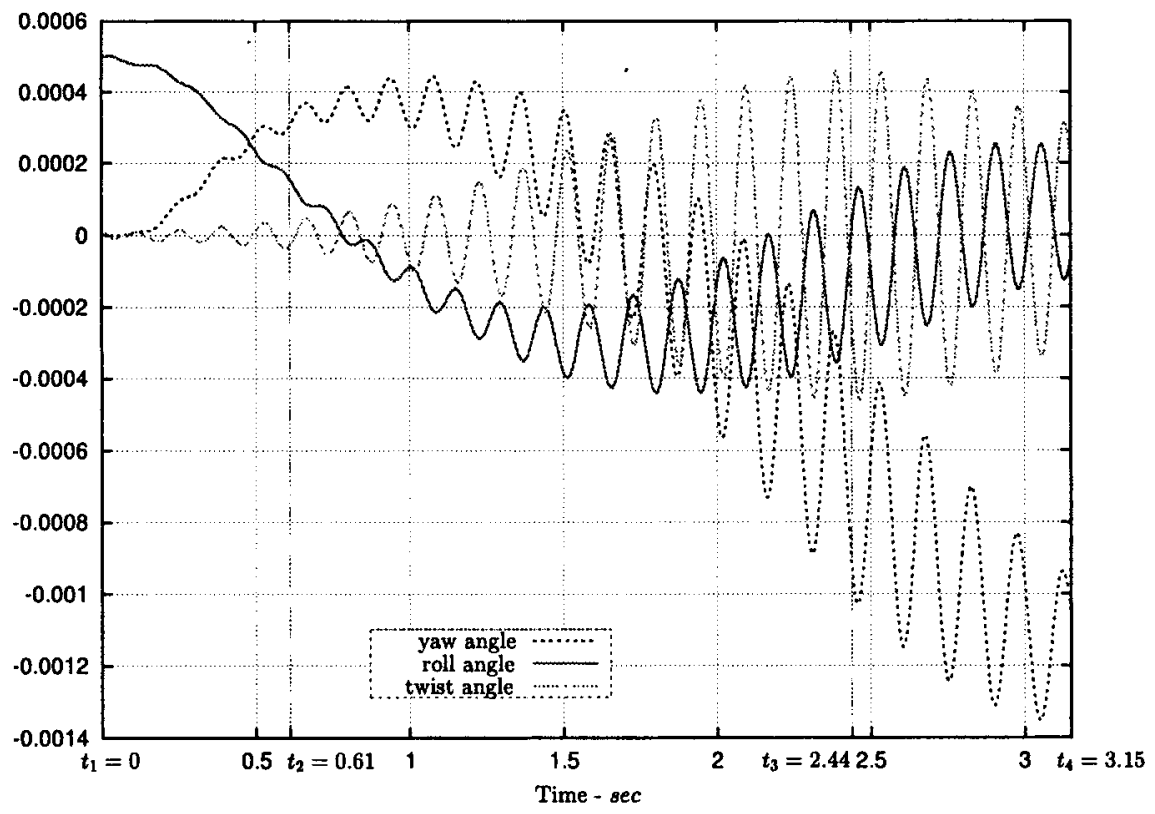

Fig. 11 Transient behaviour of the weave and wobble modes for the $2.5 \mathrm{~m} / \mathrm{s}^{2}$ deceleration case with braking 90 per cent on the front and 10 per cent on the rear wheel. The initial roll angle offset is $0.0005 \mathrm{rad}$. The time labels $t_{1}, t_{2}, t_{3}$ and $t_{4}$ can be identified in Fig. 8

On the basis of the frozen-time root-locus analysis, the wobble mode is deemed unstable at $t_{1}$, neutrally stable at $t_{2}$ and stable at times $t_{3}$ and $t_{4}$. Figure 10 shows the response of the non-linear model to a steer angle offset of $0.0001 \mathrm{rad}$ applied at time $t_{1}$. As expected, the oscillations grow until $t_{3}$ and decay thereafter. This figure is therefore in qualitative agreement with Fig. 8*. Figure 11 shows the response of the non-linear simulation model to a small roll angle offset of $0.0005 \mathrm{rad}$ that is applied at $t_{1}$ in Fig. 8. The yaw angle, roll angle and steering head twist angle all show clear evidence of both the wobble and weave modes. The high-frequency components have a frequency of roughly $7 \mathrm{~Hz}$, or $44 \mathrm{rad} / \mathrm{s}$, while the low-frequency component is of the order $2 \mathrm{rad} / \mathrm{s}$. By the time $t_{4}$ is reached, the wobble component appears to be dying out - this is most evident in the steering head twist angle. Again, these responses are all in qualitative agreement with the frozen-time linear model eigenvalue analysis. A similar set of conclusions can be drawn from Fig. 12. The only difference between Figs 11 and 12 is the braking strategy. The first figure employs correct front-wheel-dominated braking, while the second plot corresponds to incorrect rear wheel braking. Figure 13 shows the wobble mode eigenvector components corresponding to the yaw, roll and

\footnotetext{
* The first author has repeatedly noted a marked steering shimmy at about $60 \mathrm{mile} / \mathrm{h}$ under firm braking - this was not caused by disc run-out! At the time he was riding a Kawasaki ZX-9R on Snetterton race track in Norfolk and was braking down from about $140 \mathrm{mile} / \mathrm{h}$. This anecdotal evidence is in broad agreement with the theoretical results presented here.
}

twist angles. These plots show that the twist and yaw angle components are almost in phase, while the roll angle is almost in exact antiphase with the other two signals. These conclusions are in exact agreement with the phasing conclusions one derives from Fig. 11 at times $t_{3}$ and $t_{4}$.

\subsection{Braking strategies}

Every serious motorcyclist knows that the correct use of the brakes is a vital constituent of competent and safe riding. In particular, excessive use of the rear brake should never be made when braking at speed, especially if heavy braking is required in an emergency situation. This error is even more likely to end in mishap if one makes excessive use of the rear brake when banked over under cornering. In cases of mishap, the rear tyre 'lets go' and the rear end of the machine slides away resulting in a loss of control. The question is: 'Is this simply a question of rear tyre saturation, or is there a stability issue associated with these incidents as well?'

Figure 14 shows a pair of root-locus plots for the $2.5 \mathrm{~m} / \mathrm{s}^{2}$ deceleration case. In one case the front brake produces the bulk of the retarding moment, while in the other case the rear brake is used. It can be seen from this plot that braking using the front wheel has a marginally greater destabilizing effect on the wobble mode, while rear-wheel braking is to be preferred at very low speeds. The greater destabilizing effect of front braking is obvious if Figs 11 and 12 are compared. In Fig. 11, the amplitudes of the wobble mode 


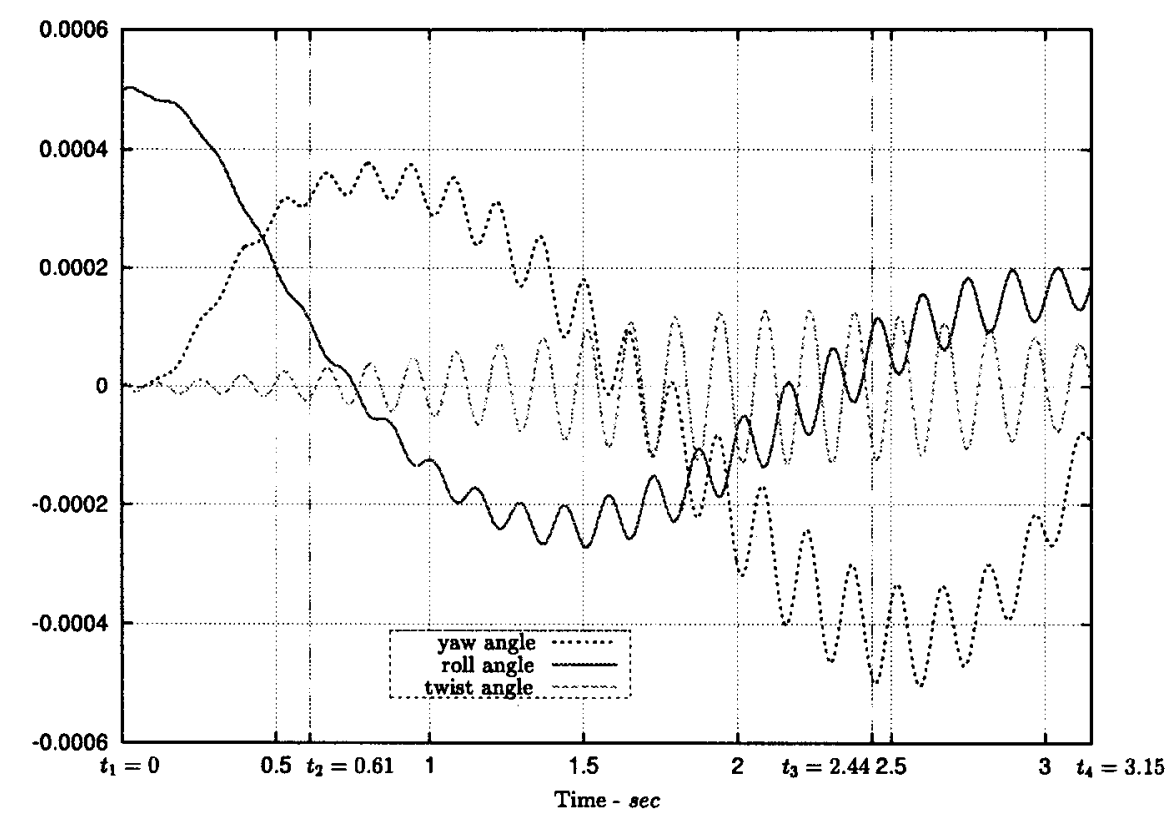

Fig. 12 Transient behaviour of the weave and wobble modes for the $2.5 \mathrm{~m} / \mathrm{s}^{2}$ deceleration case with braking 10 per cent on the front and 90 per cent on the rear. The initial roll angle offset is $0.0005 \mathrm{rad}$. The time labels $t_{1}, t_{2}, t_{3}$ and $t_{4}$ can be identified in Fig. 8

components are bigger than those in Fig. 12. The conclusion here is that the change in the braking strategy does not have a significant impact on the small amplitude machine stability.

Figure 15 examines the non-linear system behaviour under more severe rear-wheel braking at a deceleration rate of $5 \mathrm{~m} / \mathrm{s}^{2}$. It is clear from curves 1 and 2 that there is a significant load transfer from the rear tyre on to the front tyre and that this effect becomes exaggerated at lower speeds, owing to the reducing effects of aerodynamic loading.

Curves 3 and 4 show the longitudinal tyre-loading tests that are based on equation (1); the reader will recall that a tyre is deemed to have begun sliding if the associated tyre-check quantity goes positive. The front tyre-check curve is seen to go more and more negative as the speed reduces - the front tyre performs its task easily under these conditions. This reduction is attributable to the fact that the front tyre load increases as the effects of aerodynamic braking reduce. The rear tyre-check curve is both more interesting and more alarming. First it has a kink at just under $1 \mathrm{~s}$, which then goes positive at $8 \mathrm{~s}$, thereby indicating an impending mishap. The reason for the kink is as follows: at very high speed, even under deceleration, the machine has to be driven in order to overcome the effects of aerodynamic drag. At the kink, the need to drive the machine disappears and mild braking begins. Obviously, as the effects of

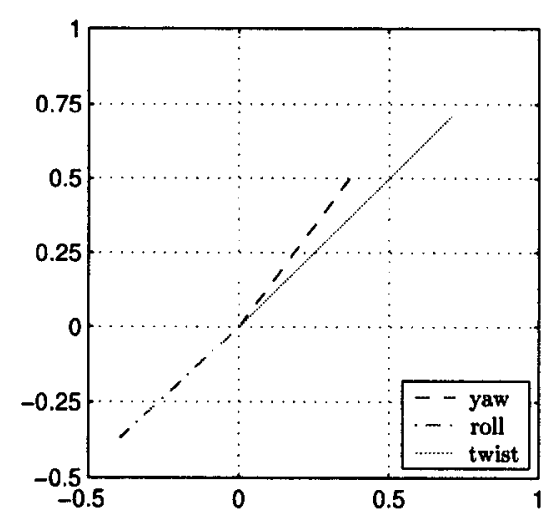

(a)

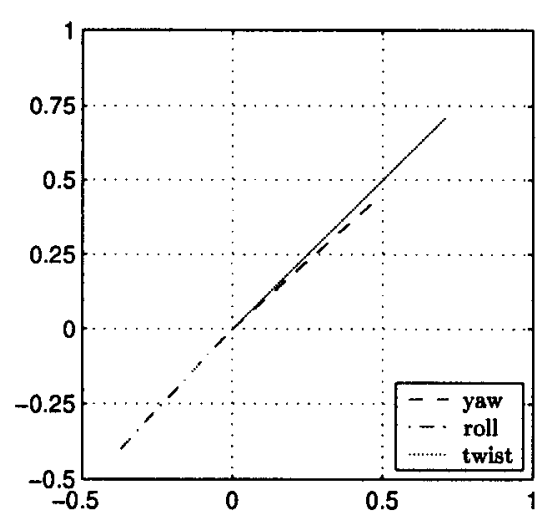

(b)

Fig. 13 Wobble-mode eigenvector components for the yaw, roll and twist angles at times (a) $t_{3}$ and (b) $t_{4}$, identified in Fig. 8 


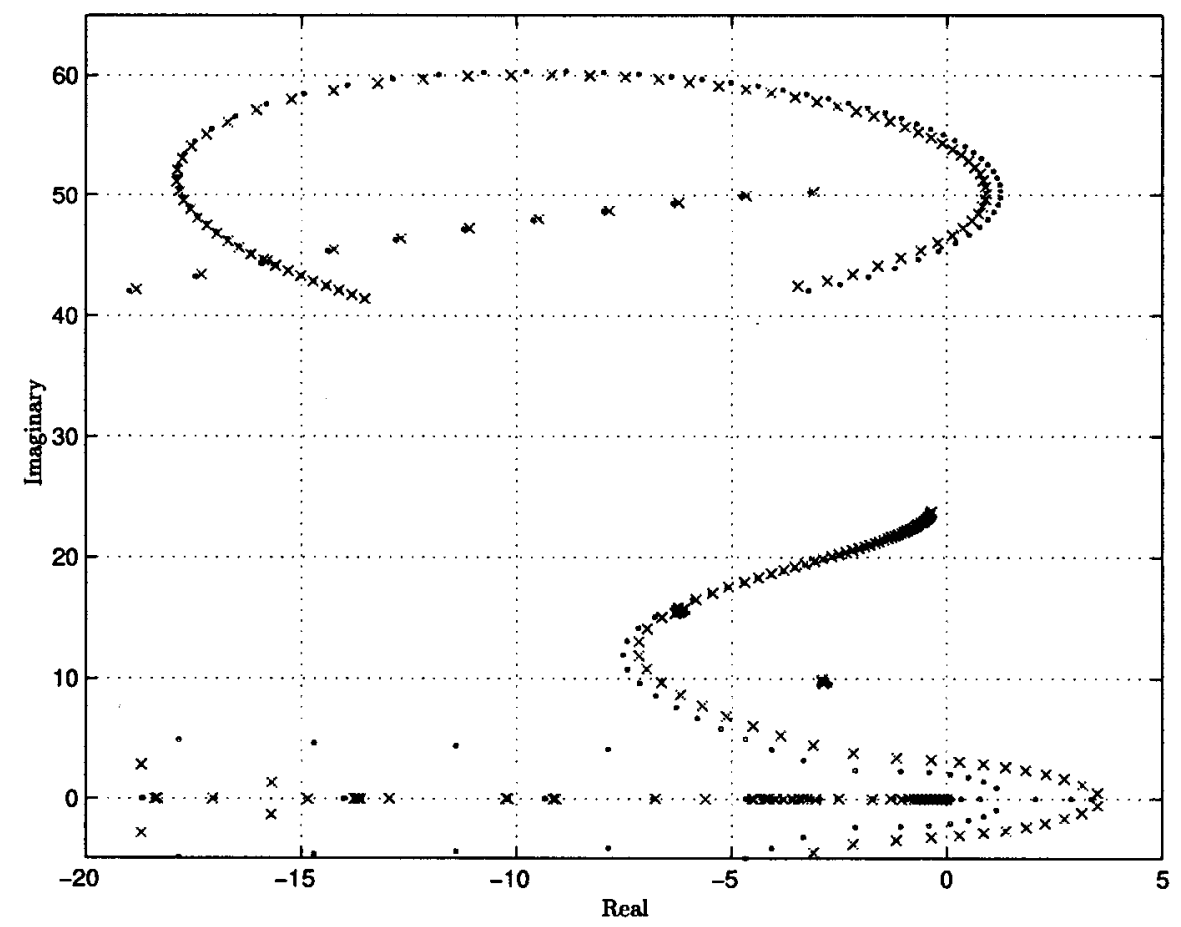

\begin{tabular}{lllll}
\hline Plot symbol & Deceleration $\left(\mathrm{m} / \mathrm{s}^{2}\right)$ & Front brake $(\%)$ & Rear brake $(\%)$ & Speed range $(\mathrm{m} / \mathrm{s})$ \\
\hline$\cdot$ & 2.5 & 90 & 10 & $70.0-0.126$ \\
$\times$ & 2.5 & 10 & 90 & $70.0-0.175$ \\
\hline
\end{tabular}

Fig. 14 Root loci for different braking conditions at a deceleration of $2.5 \mathrm{~m} / \mathrm{s}^{2}$

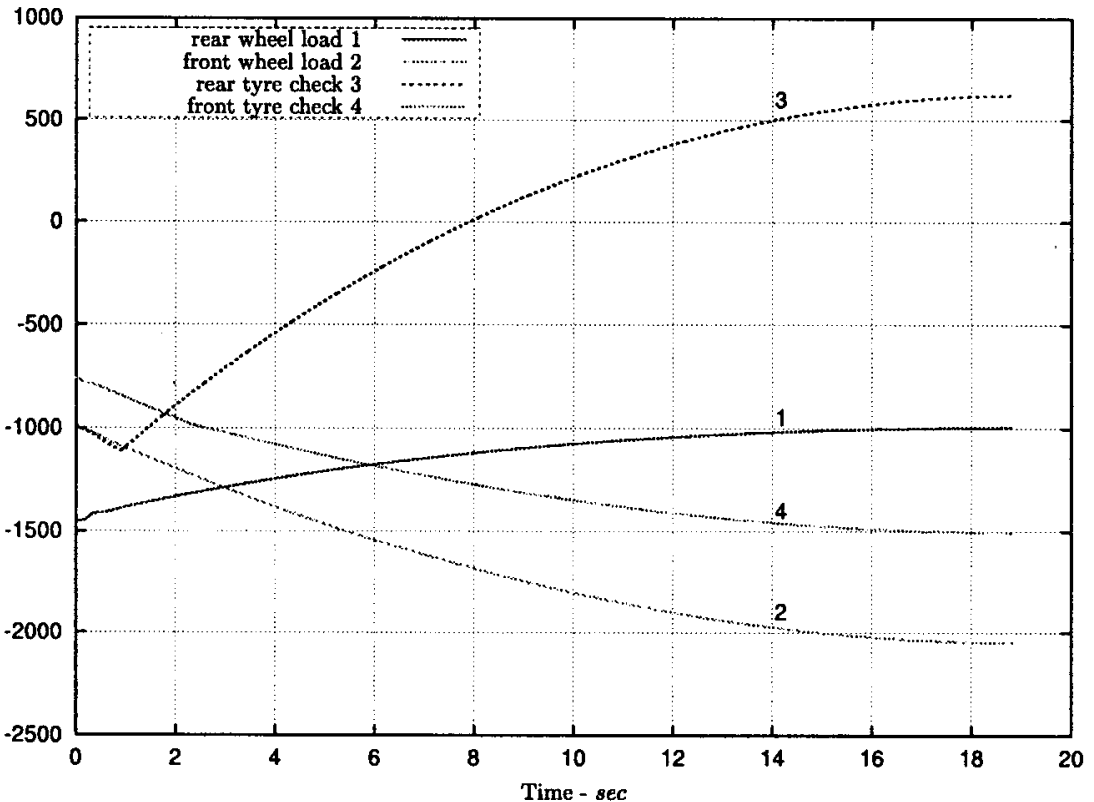

Fig. 15 Normal wheel loads and longitudinal force checks in the $5 \mathrm{~m} / \mathrm{s}^{2}$ deceleration case with 90 per cent of the braking on the rear wheel and 10 per cent on the front wheel. All the curves are given in $\mathrm{N}$ 
aerodynamic drag reduce, it becomes necessary to apply increasing levels of braking moment in order to sustain the predetermined rate of deceleration. In other words, the reducing effect of aerodynamic drag acts to undermine the rear tyre in two ways. Firstly, as this drag reduces, the brakes (especially the rear brake) have to work harder. Secondly, as the drag reduces the normal load on the rear tyre reduces, causing it to saturate. The strong well-known message is: 'heavy braking must be done on the front brake'. Under extreme track conditions, the rear brake should be used to do little more than remove the angular momentum from the rear wheel. After all, the rear tyre may become airborne in a stoppie.

\section{CONCLUSIONS}

A comprehensive study of the effects of acceleration and deceleration on motorcycle stability is presented. This work is based on a modified version of the dynamic model that was first presented in [12] and is thought to be the most elaborate motorcycle dynamic model in the public domain. Extensive use has been made of both non-linear and linearized models. The models are written in LISP and make use of the multibody modelling package AUTOSIM [10]. They are available at the web site http://www.ee.ic.ac.uk/control/motorcycles/. Interested readers are encouraged to study this code, because it contains many matters of detail that could not be covered here due to space constraints. There is some novelty in the way control systems have been used to control the motorcycle drive and braking systems in order that the machine maintains desired rates of acceleration or deceleration. The AUTOSIM code ensures that the drive moment is applied to the rear wheel, while the braking torques are shared between the two wheels according to some preassigned braking strategy. Care was taken to discount any results that were tainted by wheelies, stoppies, tyre saturation or excessive demands on engine power-this contributes to the conviction that the results presented are realistic.

The results show that the wobble mode of a motorcycle is significantly destabilized when the machine is descending an incline, or braking on a level surface. These findings have been substantiated by the first author on his own machine. This tendency of the machine to 'shake its head' is often ameliorated by fitting a steering damper. Conversely, the wobble mode damping is substantially increased when the machine is ascending an incline at constant speed or accelerating on a level surface. This probably accounts for the stable 'feel' of the machine under acceleration.

Except at very low speeds, inclines, acceleration and deceleration appear to have very little effect on the damping or frequency of the weave mode. It was reported in reference [8] that acceleration can introduce a large reduction in weave mode damping and that the weave and wobble modes can lose their identities due to a narrowing of the frequency gap between these modes. Neither of these effects was observed in this study and this discrepancy was attributed to the extreme simplicity of the model employed in reference $[\mathbf{8}]$ as well as on differing parameters.

A review of the known results on the stability of linear time-varying systems reinforces the idea that one has to be extremely careful when testing the stability of these systems via the eigenvalues of frozen-time models. This situation is especially problematic when the eigenvalues cross the imaginary axis, or are close to it. In the work presented here, the conclusions drawn from linearized frozen-time models were checked positively against nonlinear simulations.

Non-linear simulations have reinforced and quantified the known problems to do with rear tyre adhesion in heavy braking situations that are dominated by rear wheel braking. The analysis has quantified the fact that the motorcycle normal tyre loading will be transferred to the front tyre under heavy braking. Consequently, if an attempt is made to slow the machine using rear wheel dominated braking, it is very likely that the rear tyre will go into a slide, thereby inducing an irrecoverable loss of control. The aerodynamic drag acts to counteract these difficulties at high speeds.

\section{REFERENCES}

1 Sharp, R. S. The stability and control of motorcycles. J. Mech. Engng Sci., 1971, 13(5), 316-329.

2 Sharp, R. S. Vibrational modes of motorcycles and their design parameter sensitivities. In IMechE Conference on Vehicle NVH and Refinement, 1994, paper C487/016, pp. 107-121 (Mechanical Engineering Publications, London).

3 Nishimi, T., Aoki, A. and Katayama, T. Analysis of straight running stability of motorcycles. In Tenth International Technical Conference on Experimental Safety Vehicles, 15 July 1985.

4 Wier, D. H. and Zellner, J. W. Lateral-directional motorcycle dynamics and rider control. Soc. Automot. Engrs, 1979, 1364-1388.

5 Roe, G. E. and Thorpe, T. E. A solution of the low-speed wheel flutter instability of motorcycles. J. Mech. Engng Sci., 1976, 18(2), 57-65.

6 Sharp, R. S. The lateral dynamics of motorcycles and bicycles. Vehicle System Dynamics, 1985, 14, 265-283.

7 Koenen, C. The dynamic behaviour of motorcycles when running straight ahead and when cornering. $\mathrm{PhD}$ thesis, Delft University, 1983.

8 Sharp, R. S. The stability of motorcycles in acceleration and deceleration. In IMechE Conference Proceedings on Braking of Road Vehicles, 1976, pp. 45-50 (Mechanical Engineering Publications, London).

9 Hales, F. D. Lateral stability problems of simply articulated vehicles. In Proceedings of IUTAM Symposium on Recent Progress in Linear Mechanical Vibrations, 1965, pp. 17-34. 
10 Mechanical Simulation Corporation Autosim 2.5+ Reference Manual, 1998. http://www.trucksim.com.

11 Limebeer, D. J. N., Sharp, R. S. and Gani, M. R. A motorcycle model for stability and control analysis. In Euromech Colloquium 404 on Advances in Computational Multibody Dynamics (Eds J. A. C. Ambrosio and W. O. Schiehlen), 1999, pp. 287-312.

12 Sharp, R. S. and Limebeer, D. J. N. A motorcycle model for stability and control analysis. Multibody System Dynamics, 2001, 6(2) (in press).

13 Sharp, R. S. Variable geometry active rear suspension for motorcycles. In Proceedings of the 5th International Symposium on Automotive Control (AVEC 2000), Ann Arbor, Michigan, 22-24 August 2000, pp. 585-592.
14 Evangelou, S. and Limebeer, D. J. N. Lisp programming of the 'Sharp 1971' motorcycle model, 2000. http://www. ee.ic.ac.uk/control/motorcycles.

15 Evangelou, S. and Limebeer, D. J. N. Lisp programming of the 'Sharp 1994' motorcycle model, 2000. http://www. ee.ic.ac.uk/control/motorcycles.

16 Pacejka, H. B. and Sharp, R. S. Shear force development by pneumatic tyres in steady state conditions: a review of modelling aspects. Veh. System Dynamics, 1991,20, 121-176.

17 Kailath, T. Linear Systems, 1980, Ch. 12, p. 607 (PrenticeHall, Englewood Cliffs, New Jersey).

18 Desoer, C. A. Slowly varying system $\dot{x}=a(t) x$. IEEE Trans. Auto. Control, 1969, AC-14(12), 780-781.

19 Skoog, R. A. and Lau, C. G. Y. Instability of slowly varying systems. IEEE Trans. Auto. Control, 1972, AC-17(1), 86-92. 